\title{
Von Diskursen und Diskussionen
}

\author{
Andreas Klee ${ }^{1}$ Angelika Krehl ${ }^{2}$
}

Online publiziert: 20. Juli 2017

(C) Springer-Verlag GmbH Deutschland 2017

In diesem Heft von „Raumforschung und Raumordnung I Spatial Research and Planning" stellen wir einen Artikel in der Rubrik „Zur Diskussion/Statement“ vor, der ein in der Wissenschaft und noch viel mehr in der beruflichen Praxis spürbares Unbehagen aufgreift. Worum geht es? In den letzten Jahren hat sich in den Raumwissenschaften, insbesondere in der Humangeographie, ein Forschungsansatz etabliert, der scheinbare Gewissheiten ins Wanken gebracht hat. Der Sozialkonstruktivismus, demzufolge - kurz gesagt - die soziale Wirklichkeit „konstruiert“ und nicht als gegeben, gesichert und objektiv nachprüfbar erachtet wird, ist die konzeptionelle und methodologische Grundlage vieler neuerer Forschungsarbeiten. Die soziale Wirklichkeit wird in diesem Verständnis als in ständiger Veränderung begriffen, die Menschen reproduzieren sie über ihre Vorstellungen, Intentionen und Handlungsweisen permanent. Um zu begreifen, auf welche Art und Weise individuelle Konstruktionen erfolgen, welche Bedeutungen sie haben und welche Handlungen sie möglicherweise implizieren, wird häufig auf die Methode der Diskursanalyse zurückgegriffen. Der hier zugrunde liegende Diskursbegriff wird in der Regel verstanden als ,,überindividuelle Muster des Denkens, Sprechens [...] und Handelns sowie Prozesse, in denen bestimmte Vorstellungen und Handlungslogiken

Prof. Dr. Andreas Klee

klee@arl-net.de

Angelika Krehl

angelika.krehl@ils-forschung.de

1 Akademie für Raumforschung und Landesplanung, Leibniz-Forum für Raumwissenschaften, Hohenzollernstraße 11, 30161 Hannover, Deutschland

2 ILS - Institut für Landes- und Stadtentwicklungsforschung, Brüderweg 22-24, 44135 Dortmund, Deutschland hergestellt und immer wieder verändert werden“ (Glasze/ Mattissek 2009: 11 f.). Diese Muster gilt es in der Diskursanalyse nachzuzeichnen, weniger zu erklären.

Dies ist sicherlich nicht die richtige Stelle für eine tiefgehende und wissenschaftlich korrekte Auseinandersetzung mit dem Sozialkonstruktivismus, seinen Potenzialen und Grenzen. Aber er bewegt die Wissenschaftlerinnen und Wissenschaftler. Bei genauem Hinsehen lässt sich vielfach Unverständnis für diesen Forschungsansatz, manchmal geradezu ein Unbehagen feststellen. Hiervon handelt der Artikel von Gerd Lintz mit dem Titel „Foucault statt Fürst? Gedanken zu einem an Bedeutung gewinnenden Paradigma“. Seine Gedanken fokussieren auf die zunehmende Verwendung sozialkonstruktivistischer Ansätze in der Landschaftsforschung und das dadurch ausgelöste Unbehagen oder auch Unverständnis gegenüber diesen Ansätzen bei Wissenschaftlerinnen und Wissenschaftlern, die „klassischen“ Methodologien folgen, beispielsweise dem Kritischen Rationalismus oder gesellschaftskritischen Ansätzen. Zudem argumentiert Lintz, dass viele Praktikerinnen und Praktiker befürchten, „die konstruktivistischdiskursanalytische Wissenschaft“ entferne sich ,zu weit von den gesellschaftlichen Problemen [...] und [sei] für die alltägliche Planungspraxis nicht mehr relevant“. Er plädiert unter anderem für ein stärkeres wechselseitiges Verständnis für die unterschiedlichen wissenschaftlichen Erkenntnisinteressen sowie Ansätze der Forschenden einerseits und für die Notwendigkeit, in der Praxis vorliegende Probleme zu lösen, andererseits. Lintz' Beitrag zeigt aber auch deutlich, dass wir in den Raumwissenschaften weit davon entfernt sind, bestimmte wissenschaftstheoretische Positionen, die derzeit vermehrt angewandt werden, als „etabliert“ anzusehen. Vielmehr ist - aus Sicht der Forschenden - wohl ein ständiges Hinterfragen des eigenen Ansatzes sowie 
das Zulassen und kritische Auseinandersetzen mit anderen Ansätzen notwendig.

Welche weiteren Fragen werden in diesem Heft behandelt? Anne Vogelpohl beschäftigt sich in ihrem Beitrag mit dem doppelten Ursprung der Stadtentwicklungsstrategie „Leitbild: Metropole Hamburg - Wachsende Stadt“ sowie dem Einfluss von Unternehmensberatungen (Stichwort McKinseyisierung) auf die (strategische) Stadt- und Regionalentwicklung in Hamburg. Sie analysiert die „Ursprünge" für umfassende Stadtentwicklungsstrategien der jüngeren Zeit in deutschen Städten, die einem Effizienzgedanken folgen. Weiterhin klärt sie die Rollenverteilung von Unternehmensberatungen und Akteuren der Politik bei der Entwicklung entsprechender Strategien am Beispiel des Leitbildes „Wachsende Stadt“ für Hamburg. Vogelpohl kann resümierend zwei Hauptmerkmale einer „McKinseyisierung“ der Stadtpolitik differenzieren: die Produktion eines Policy-Kontextes über die Kanalisierung politischer Inhalte und die Produktion eines Politics-Kontextes über die Vernetzung urbaner Eliten.

Der Beitrag „Verkehrs-, umwelt- und raumbezogene Aspekte der Elektromobilität aus Sicht des Nutzerverhaltens" von Thomas Döring und Birgit Aigner-Walder untersucht die Verbreitung von elektrisch betriebenen Fahrzeugen und deren Auswirkungen auf Verkehr, Umwelt und Raum unter expliziter Berücksichtigung des bisher in der Literatur eher vernachlässigten individuellen Entscheidungsverhaltens. Diese (vermeintliche) Forschungslücke adressieren die Autoren durch eine Betrachtung der Opportunitätskosten, die mit der Nutzung von E-Fahrzeugen einhergehen. Daraus leiten sie einige Implikationen für den Verkehrsbereich, die Umwelt und eine Einschätzung über die mögliche Verbreitung von Elektrofahrzeugen im urbanen und ländlichen Raum ab.

Ein weiterer Beitrag im Themenfeld Mobilität kommt von Julia Jarass und Rebekka Oostendorp zum Thema „Intermodal, urban, mobil - Charakterisierung intermodaler Wege und Nutzer am Beispiel Berlin“. Im Anschluss an eine vertiefte Auseinandersetzung mit dem Intermodalitätsbegriff wird intermodales Mobilitätsverhalten, verstanden als die Kombination mehrerer Verkehrsmittel im Verlauf eines Weges, im Hinblick auf die verkehrsmittelspezifische Charakterisierung intermodaler Wege, die spezifischen soziodemographischen Merkmale intermodaler Personengruppen und die räumliche Verortung intermodaler Verhaltensweisen untersucht. Der Beitrag stellt einen interessanten Ansatz dar, intermodales Verhalten besser zu verstehen, und kommt zu spannenden empirischen Ergebnissen bezüglich der Verkehrsmittelwahl, der Wegelängen sowie gruppenspezifischer Besonderheiten.

Heinrich Mäding nimmt in seinem Beitrag eine fachgeschichtliche Beurteilung des 1961 veröffentlichten Gutach- tens „Die Raumordnung in der Bundesrepublik Deutschland" des Sachverständigenausschusses für Raumordnung (sogenanntes SARO-Gutachten) vor. Er misst dem Gutachten insofern eine große Leistung in der Geschichte der Raumordnung in Deutschland bei, als die Verfasser eine - aus heutiger Sicht nicht selbstverständliche - Funktionsbestimmung der Raumordnung in der Marktwirtschaft vornahmen und Richtlinien für die Politik erarbeiteten. Das Gutachten hat nach Mädings Auffassung die Verabschiedung des Raumordnungsgesetzes 1965 überhaupt erst möglich gemacht. Er bewertet es als ein frühes Beispiel für gelungene wissenschaftliche Beratung in den Jahren des Planungsoptimismus, während sein fachwissenschaftlicher Beitrag eher bescheiden genannt werden müsse. Mäding kommt zum Schluss, dass trotz vieler sachlicher Lücken, argumentativer Mängel und normativer Unausgewogenheiten des Gutachtens der Sachverständigenausschuss für Raumordnung (SARO) große Anerkennung verdiene.

Schließlich thematisieren Peter Wirth und Markus Leibenath in ihrem Artikel „Die Rolle der Regionalplanung im Umgang mit Windenergiekonflikten in Deutschland und Perspektiven für die raumbezogene Forschung" die Potenziale und Grenzen der Regionalplanung im Umgang mit Konflikten um die Errichtung von Windenergieanlagen. Sie ziehen hierzu die Fortschreibung des Regionalplans für die sächsische Planungsregion Oberes Elbtal/Osterzgebirge als Fallbeispiel heran. Von besonderem Interesse ist in diesem Zusammenhang ein speziell für diesen Zweck eingerichteter Arbeitskreis Windenergie, der Befürworter und Gegner der Windenergie an einen Tisch bringen soll. Die Ergebnisse von Wirth und Leibenath zeigen, dass die Regionalplanung einen Beitrag zur Verfahrenstransparenz leisten kann, den Konflikt zwischen Befürwortern und Gegnern der Windenergieanlagen aber letztlich nicht lösen kann.

Diskurse und Diskussionen - in diesem Heft von „Raumforschung und Raumordnung I Spatial Research and Planning" haben sich Autorinnen und Autoren aus ganz unterschiedlichen Motivationen diesem Thema angenommen und unterschiedliche Perspektiven aufgezeigt. Das Spektrum reicht dabei von grundsätzlichen Überlegungen zur Diskursanalyse bis hin zum praxisnahen Umgang mit Konflikten in Arbeitskreisen.

Wir wünschen allen Leserinnen und Lesern eine anregende Lektüre.

\section{Literatur}

Glasze, G.; Mattissek, A. (2009): Diskursforschung in der Humangeographie: Konzeptionelle Grundlagen und empirische Operationalisierungen. In: Glasze, G.; Mattissek, A. (Hrsg.): Handbuch Diskurs und Raum. Theorien und Methoden für die Humangeographie sowie die sozial- und kulturwissenschaftliche Raumforschung. Bielefeld, 11-59. 